\title{
Development of a Structure-Validated Nightmare Experience Questionnaire in Chinese University Students
}

\author{
Wanzhen Chen ${ }^{1,2}$, You Xu ${ }^{1}$, Ming Zhu ${ }^{1}$, Yixin Tang ${ }^{1}$, Shenyu Huang ${ }^{1}$, Hongjing Mao ${ }^{3}$, Jian Liu ${ }^{3}$ and Wei Wang ${ }^{1,2,3}$ \\ ${ }^{1}$ Department of Clinical Psychology and Psychiatry/School of Public Health, Zhejiang University College of Medicine, Hangzhou, China \\ ${ }^{2}$ Department of Psychology and Behavioral Sciences, Zhejiang University College of Sciences, Hangzhou, China \\ ${ }^{3}$ Department of Clinical Psychology, Affiliated Psychiatric Hospital, Zhejiang University College of Medicine, Hangzhou, China
}

"Corresponding author: Wei Wang, Department of Clinical Psychology and Psychiatry/School of Public Health, Zhejiang University College of Medicine, Yuhangtang Road 866, Hangzhou, Zhejiang 310058, China,Tel: +86-571-88208188; E-mail: wangmufan@msn.com

Received Date: July 16, 2014, Accepted Date: September 23, 2014, Published Date: September 30, 2014

Copyright: ( ) 2014, Wei Wang et al., This is an open-access article distributed under the terms of the Creative Commons Attribution License, which permits unrestricted use, distribution, and reproduction in any medium, provided the original author and source are credited.

\begin{abstract}
Background: Nightmare experience is associated with a broad spectrum of psychopathology, however, there lacks a structure-validated questionnaire to measure the effects of nightmare contents on the physical, psychological and behavioral aspects.

Methods: Based on the clinical manifestation and previous documentation, we have designed a matrix of 95 items to measure the nightmare contents, plus one item to measure the nightmare frequency, and trialed them in 160 male and 161 female Chinese university students who have had a life-long nightmare experience.

Results: After both exploratory and confirmatory factor analyses, we have found four factors regarding the nightmare experience, namely Physical Effect, Negative Emotion, Meaning Interpretation and Horrible Stimulation. The fit of 20-item model (five top-loading items each factor) has proven to be the best structure. Together with an item measuring frequency, the model, called Nightmare Experience Questionnaire (NEQ), was retained for further analyses. The nightmare frequency was positively correlated with all the four NEQ factors, and women scored significantly higher on NEQ Negative Emotion and Meaning Interpretation factors than men did.
\end{abstract}

Conclusion: The NEQ might help to understand some psychiatric disorders, especially those with the awaking emotional deregulation in women.

Keywords: Confirmatory factor analysis; Nightmare experience; Principal component analysis; Structure-validated

\section{Introduction}

Nightmares are repeated occurrences of extended, extremely dysphoric, and well-remembered dreams usually involving efforts to avoid threats to survival, security, or physical integrity, and usually being accompanied by fear or panic which results in immediate awakening [1]. An individual generally has a nightmare during the rapid eye movement sleep, and quickly becomes oriented and alert on waking from the dream; however, the nightmare might leave the individual negative emotions and cause obvious distress, emotional deregulation, or impairment in social, occupational, or other functioning [2,3]. The life-long nightmare experience happens in almost every healthy individual $[4,5]$, especially in adolescents and young adults, or in women [6,7].

In clinics, the frequent nightmares are prevalent in $16.7 \%$ patients with schizophrenia [8], 17.5\% depression [8,9], 25\% substance or alcohol abuse [10,11], 49\% borderline personality disorder [12,13], 57\% dissociative disorder $[14,15]$, and $90 \%$ post-traumatic stress disorder (PTSD) [16]. Moreover, the frequent nightmares happen in anxiety and bipolar disorders $[17,18]$. They are even considered as a hallmark of PTSD [3,9], since they are connected with a broad range of traumatic events, such as combat exposure, traffic accident, crime victimization, rape, and natural disaster [20-23]. There is a robust association between the nightmare frequency and the severity of the clinical symptom of the psychiatric disorders mentioned-above [3]. In addition, the frequent nightmares in early adolescence predict the onset of anxiety symptoms five years later [18], and increase the suicidal tendency in patients with major depression, borderline personality disorder, and dissociative disorder [24,25].

Scholars have developed several inventories to measure the frequency and different aspects of nightmare. For instance, there are one questionnaire measuring the nightmare effects of distress: the 13item Nightmare Distress Questionnaire (with an internal alpha of between 0.83 and 0.88 ) [26]; one questionnaire measuring its effects on emotion: the 4-item Nightmare Emotions Scale (with an internal alpha of 0.62) [27]; one questionnaire on daily behaviors: the 43-item Nightmare Behavior Questionnaire [28]; and two questionnaires on daily activities: the 11-item Nightmare Effects Survey [29] and the 13item Nightmare History and Impact Questionnaire [30]. Although the Nightmare Distress Questionnaire has proven to be a three-factor structured one (i.e., preoccupation-fear, interference, and premonition), and the Nightmare Effects Survey a two-factor one (specific effects, and general effects) [31], most of these questionnaires were not structurally-validated with a structure-modeling equation 
Page 2 of 6

method. Moreover, up to present, there is no single study using an overall self-report questionnaire for nightmare regarding the dream contents and their effects on the cognitive, physical, and behavioral aspects of the individual.

The aim of the present study was to develop a structure-validated measurement of nightmare experience which might cover different nightmare topics, the experienced emotion, the cognition and behavior afterwards, and the physical/ psychological effects. Inspired by previous studies $[26,28,29,32,33]$ and the definition of the nightmare in DSM-5 [1], we have built a matrix of statements regarding the nightmare experience. We have hypothesized that: (1) the nightmare experience contents are clearly divided into: the nightmare topic, the emotion during dreaming and subsequent waking states, the cognition, behavior and physical/ psychological functions during waking state afterwards; (2) the nightmare frequency is correlated with these content measures; (3) women display pronounced emotional and behavioral effects which relating to the nightmares.

\section{Methods}

\section{Participants}

Totally 354 participants were invited to participate in the current study. Participants who never had a nightmare experience $(n=23)$ or had not responded to the 95-item matrix measuring nightmares (see below) $(\mathrm{n}=10)$ were excluded. The remaining participants were 160 men (mean age: 20.13 years \pm 1.12 S.D.; range: $18-25$ years) and 161 women (mean age: $19.97 \pm 1.29$; range: 18-29). There was no significant age difference between the two gender groups (the Student $\mathrm{t}=1.12, \mathrm{p}=0.26,95 \%$ Confidence Interval (CI): $-0.12 \sim 0.42$ ). They were all undergraduates of Han ethnicity, and majored in Arts, Education, Foreign Languages, Engineering, Math, Mechanics, and Modern Medicine. All participants were free from any somatic or psychiatric illnesses (including sleep-wake disorder and PTSD), had not experienced recent life-events, and had not received horror video, picture or sound stimulation at least 72 hours prior to participating in the study. The study protocol was approved by a local Ethics Committee and all participants had given their written informed consent. Moreover, the participants were paid or earned course credits for their participation.

\section{Measures}

Participants were asked to complete a matrix of 95 items measuring the nightmare experience in a quiet room. They were asked to use a five-point Likert rating scale: 1-very unlike me, 2-moderately unlike me, 3-somewhat like and unlike me, 4-moderately like me, and 5-very like me. The main parts of the items were: 1) negative emotion experience in nightmare, 2) aggressivity or grievous bodily harm in nightmare, 3) events in nightmare, 4) dream recall clarity of nightmare, 5) behavior and cognition after nightmare, 6) psychological and physiological effects after nightmare (including the immediate emotion of waking from nightmare). An additional item measuring the nightmare frequency was also adopted from a previous study [32], and participants were asked to rate it by an eight-point Likert rating scale, i.e., 1-never, 2-less than once a year, 3-once a year, 4-two-to-four times a year, 5-once a month, 6-two-to-three times a month, 7-once a week, and 8 -several times a week.

\section{Statistical analyses}

Answers to the 95 items were subjected to the principal component analysis using the Predictive Analytics Software Statistics, Release Version 18.0.0 (SPSS Inc., 2009, Chicago, IL) [34]. The factor loadings were rotated orthogonally using the varimax normalized methods. Items which were loaded less heavily (below 0.45 ) on a target factor, or cross-loaded heavily (above 0.30 ) on more than one factors were removed from subsequent analyses one-by-one.

The fit of the remaining data (i.e., components extracted as latent factors) thereafter were evaluated by the confirmatory factor analysis for the structural equation modeling using the Analysis of Moment Structures (AMOS), version 17.0 (Arbuckle, AMOS Development Corp. 2008, Crawfordville, FL) [35]. Indices used to assess the overall fit model were the $\chi^{2} / \mathrm{df}$, the goodness of fit index [36], the adjusted goodness of fit index, the comparative fit index [37], the Tucker-Lewis Index [38], and the root mean square error of approximation [39].

Once factors and the related items were identified, their scores were calculated in each gender group. The internal reliabilities (the Cronbach alphas) for each factor were also calculated in all participants by another computer program - Reliability and Item Analysis. The gender difference of individual factor scores was evaluated by two-way ANOVA (i.e., gender $\times$ factor score) plus the independent Student $t$ test, and the gender difference of the nightmare frequency was analyzed by the Mann-Whitney $U$ test. Moreover, the Pearson correlation test was used to search for possible relationships between the frequency and the four factors, and for those within the factors. A p value less than 0.05 was considered to be significant.

\section{Results}

Answers to the 95 items measuring the nightmare experience were entered into a principal component analysis first. Results of the preanalysis check were acceptable $(\mathrm{KMO}=0.897$; the Bartlett test of sphericity=15588.39; $\mathrm{p}=0.000$ ). Twenty-five eigen values greater than 1.0 were identified, and the scree plot indicated a level-off from the sixth factor on. The eigen values of the first four factors were 14.16, 8.36, 5.64 and 5.54 respectively, which accounted for $35.58 \%$ of the total variance. After the varimax normalized rotation, there were less than three items loaded appropriately on each of the fifth and the sixth factor. Therefore, a four-factor solution was chosen for the confirmatory factor analysis, with 40 out of 95 items (19,8,7,6 items respectively for the four factors) which loaded higher than 0.45 on the target factor and not cross-loaded heavily on other factors.

Six AMOS fit-models of four-factors with different items were constructed (Table1) and the 20-item model (five items with top loadings on each target factor) was the best among them. The standardized factor correlations for the 20-item model structure were also acceptable (Figure 1). Based on these 20 items, and an item measuring the nightmare frequency, we have developed a Nightmare Experience Questionnaire (NEQ, Table 2), and consequently named its four factors as below.

Factor 1 was called "Physical Effect", which reflects a deficit of the physical health, appetite, and other daily activities after nightmares; the higher score the higher level of physical impairment. Factor 2 was called "Negative Emotion", which describes frightened, scared, helpless emotions during nightmares; the higher score the more negative emotion experienced. 
Citation: Chen W, Xu Y, Zhu M, Tang Y, Huang S, et al. (2014) Development of a structure-validated Nightmare Experience Questionnaire in
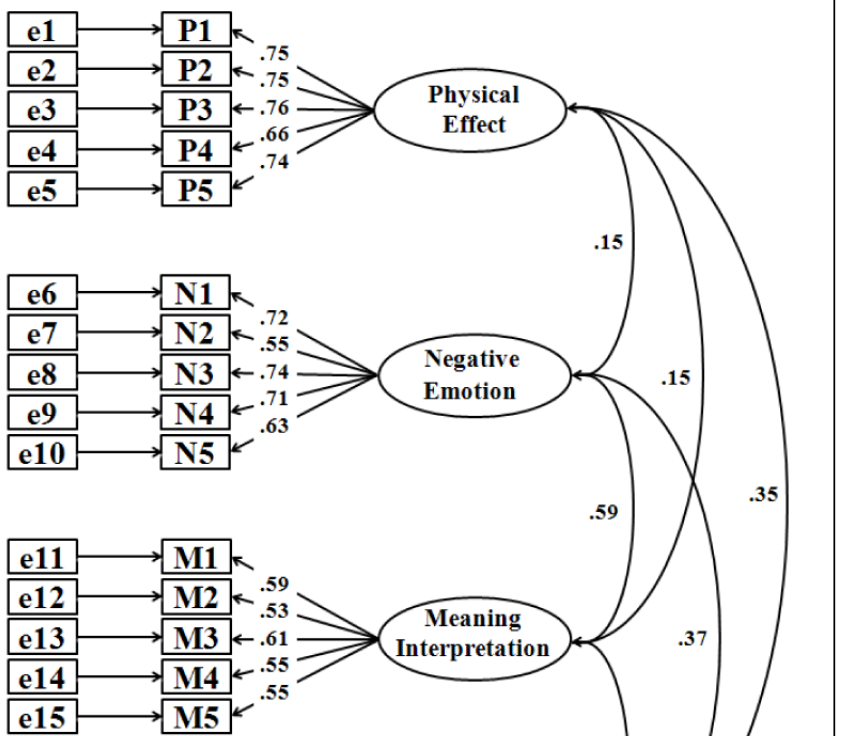

$$
\text { e15 }
$$$$
\rightarrow \text { M5 }
$$
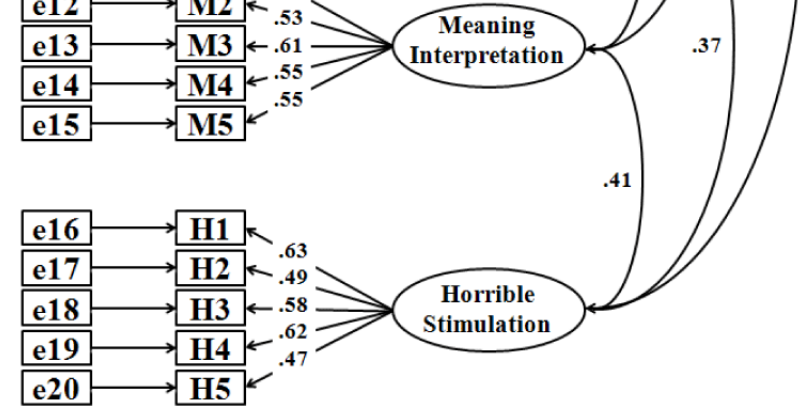

Figure 1: Standardized factor structures for a confirmatory factor analysis of a four-factor Nightmare Experience Questionnaire (NEQ) model.
Factor 3 was called "Meaning Interpretation", which describes an effort to interpret the nightmare or to value the information it might convey; the higher score the more rumination evoked. Factor 4 was called "Horrible Stimulation", which describes scenes of terrifying activity or adventure, violence, or terrorist incidents; the higher score the more horror or excitation experienced.

Two-way ANOVA detected a significant difference in the four NEQ factor scores between two gender groups $(F[1,319]=4.94, p=0.027$, mean squared effect=133.58). The post-hoc Student t test showed that the scores of NEQ Negative Emotion and Meaning Interpretation were significantly higher $(\mathrm{p}<0.05)$ in women than those in men. The internal alphas of the four factors were satisfactory, and the intercorrelations between the four factors were significant but remained in a low or medium level (Table 3 ).

For the nightmare frequency, nine participants failed to provide an answer. In the rest 312 participants who had a life-long nightmare experience, about 61 people (19\%) reported a frequency of less than once a year, $30(9.3 \%)$ once a year, $138(43 \%)$ of two-to-four times a year, $37(11.5 \%)$ once a month, $36(11.2 \%)$ two-to-three times a month, $5(1.6 \%)$ once a week, and $5(1.6 \%)$ several times a week. The Mann-Whitney $U$ test failed to detect a gender difference $(\mathrm{U}=12063.00, \mathrm{p}=0.89)$ regarding the nightmare frequency. There were however, significant positive correlations between nightmare frequency and NEQ Physical Effect $(n=312, r=0.21)$, Negative Emotion (0.26), Meaning Interpretation (0.27) and Horrible Stimulation (0.26) factors.

\begin{tabular}{|l|l|l|l|l|l|l|l|}
\hline $\begin{array}{l}\text { Total item } \\
\text { number }\end{array}$ & $\begin{array}{l}\text { Item numbers } \\
\text { each factor }\end{array}$ & $\mathbf{X}$ 2/df & $\begin{array}{l}\text { Goodness of } \\
\text { fit index }\end{array}$ & $\begin{array}{l}\text { Adjusted } \\
\text { goodness of fit } \\
\text { index }\end{array}$ & $\begin{array}{l}\text { Comparative fit } \\
\text { index }\end{array}$ & $\begin{array}{l}\text { Tucker-Lewis } \\
\text { index }\end{array}$ & $\begin{array}{l}\text { Root mean square error of } \\
\text { approximation }\end{array}$ \\
\hline 40 & $19,8,7,6$ & 2.16 & 0.79 & 0.76 & 0.80 & 0.79 & 0.060 \\
\hline 31 & $10,8,7,6$ & 2.03 & 0.85 & 0.83 & 0.86 & 0.85 & 0.057 \\
\hline 29 & $8,8,7,6$ & 2.12 & 0.85 & 0.83 & 0.87 & 0.84 & 0.059 \\
\hline 24 & $6,6,6,6$ & 2.07 & 0.89 & 0.86 & 0.88 & 0.87 & 0.058 \\
\hline 23 & $6,6,6,5$ & 2.19 & 0.88 & 0.86 & 0.88 & 0.86 & 0.061 \\
\hline 20 & $5,5,5,5$ & 2.00 & 0.91 & 0.89 & 0.91 & 0.89 & 0.056 \\
\hline
\end{tabular}

Table 1: Four-factor fitting models of the Nightmare Experience Questionnaire in 321 participants.

\section{Discussion}

Using both exploratory and confirmatory factor analyses on the 95 items regarding nightmare, we have developed a structure-validated, 20-item Nightmare Experience Questionnaire (NEQ) in Chinese university students. The four factors namely Physical Effect, Negative Emotion, Meaning Interpretation, and Horrible Stimulation, had satisfactory internal reliabilities and low-to-medium intercorrelations, which supported our first hypothesis. The $3.2 \%$ prevalence of high frequency-nightmare (more than once a week) in our participants was similar to the $2-6 \%$ prevalence reported earlier [40]. The significant correlations between nightmare frequency and four NEQ factor scores, and the women preponderance in NEQ Negative Emotion and Meaning Interpretation factors supported other two of our hypotheses. Previous documentation tells that the nightmare frequency is related to how individuals view their dreams in general [3]; our results have demonstrated further that it was related to four distinctive aspects of nightmare experience in detail. 
Citation: Chen W, Xu Y, Zhu M, Tang Y, Huang S, et al. (2014) Development of a structure-validated Nightmare Experience Questionnaire in Chinese University Students. J Psychiatry 17: 1000147. doi:10.4172/2378-5756.1000147

Page 4 of 6

The first factor, Physical Effect, was a replication of the nightmare distress [26], of the physiological effects $[29,30]$ and of the behavioral effects [28] reported previously. Indeed, the chronic nightmare sufferers often attributed their daytime impairment to the increased fatigue from the related poor night-sleep [41]. The positive correlation between the nightmare frequency and the Physical Effect score found in our study was also in line with a previous report [42].

\begin{tabular}{|c|c|c|c|c|}
\hline Items & Factor 1 & 2 & 3 & 4 \\
\hline I feel getting weak physically because of having nightmares. & 0.71 & -0.03 & 0.01 & 0.10 \\
\hline My appetite has changed because of having nightmares. & 0.70 & -0.06 & -.05 & 0.15 \\
\hline I find that I cannot deal with activities the day after having nightmares. & 0.69 & -0.05 & -.04 & 0.21 \\
\hline I always suffer from insomnia due to having nightmares. & 0.68 & 0.07 & 0.04 & 0.15 \\
\hline I always consider that my life is deeply influenced by nightmares. & 0.64 & -0.07 & 0.06 & 0.08 \\
\hline I felt frightened in my nightmares. & 0.05 & 0.67 & 0.16 & -.01 \\
\hline I was scared in my nightmares. & 0.05 & 0.63 & 0.19 & 0.10 \\
\hline I often feel helpless in my nightmares. & 0.07 & 0.63 & 0.23 & 0.09 \\
\hline I can hardly get out of the dilemma in my nightmares. & -0.02 & 0.60 & 0.14 & 0.27 \\
\hline I can do nothing to help facing the occurrence in my nightmares. & 0.06 & 0.54 & 0.14 & 0.13 \\
\hline I try to interpret the contents of nightmare. & 0.19 & 0.12 & 0.61 & 0.13 \\
\hline Nightmares make no sense to me. & -0.18 & 0.00 & -0.55 & 0.20 \\
\hline Nightmares contain some important information which I am very cautious about. & 0.18 & 0.23 & 0.55 & 0.02 \\
\hline I can always see the details clearly in my nightmares. & -0.05 & 0.26 & 0.53 & 0.18 \\
\hline The scene or person involved in my nightmares was familiar to me. & -0.07 & 0.19 & 0.49 & 0.13 \\
\hline I dreamed about participating in a terrifying activity or adventure. & 0.10 & 0.10 & 0.18 & 0.60 \\
\hline I feel excited in my nightmares. & 0.22 & -0.07 & 0.01 & 0.54 \\
\hline My nightmares always go along with violence. & 0.15 & 0.26 & -0.04 & 0.53 \\
\hline I often dream about the terrorist incidents that threaten lives of others. & 0.15 & .23 & 0.18 & 0.49 \\
\hline In my nightmares, I dreamed about doing something I never do in my daily routines. & 0.05 & 0.05 & 0.05 & 0.47 \\
\hline
\end{tabular}

Table 2: Factor loadings of the selected 20 items after the principal component analysis and confirmatory factor analysis in 321 participants. Note: Loadings $\geq 0.45$ are in bold for clarify.

\begin{tabular}{|l|l|l|l|l|l|l|l|}
\hline \multicolumn{2}{|l|}{} & \multicolumn{2}{|l|}{ Factor Score } & $\begin{array}{l}\text { Alpha } \\
(\mathbf{n}=\mathbf{3 2 1})\end{array}$ & \multicolumn{2}{l|}{ Inter-correlation $(\mathbf{n = 3 2 1 )}$} \\
\hline & $\begin{array}{l}\text { Men } \\
(\mathbf{n}=\mathbf{1 6 0})\end{array}$ & $\begin{array}{l}\text { Women } \\
(\mathbf{n}=\mathbf{1 6 1 )}\end{array}$ & $\mathbf{9 5 \%} \mathbf{C l}$ & & $\begin{array}{l}\text { Physical } \\
\text { Effect }\end{array}$ & $\begin{array}{l}\text { Negative } \\
\text { emotion }\end{array}$ & $\begin{array}{l}\text { Meaning } \\
\text { interpretation }\end{array}$ \\
\hline Physical effect & $7.16 \pm 3.37$ & $6.71 \pm 2.53$ & $-0.20 \sim 1.11$ & 0.85 & & & \\
\hline Negative emotion & $16.26 \pm 4.47$ & $17.89 \pm 4.14 \#$ & $-2.58 \sim-0.69$ & 0.80 & $0.13^{*}$ & & \\
\hline Meaning interpretation & $13.21 \pm 3.97$ & $15.04 \pm 4.02 \#$ & $-2.72 \sim-0.96$ & 0.65 & $0.14^{*}$ & $0.42^{*}$ & \\
\hline Horrible stimulation & $12.94 \pm 4.24$ & $12.50 \pm 4.07$ & $-0.47 \sim 1.35$ & 0.69 & $0.28^{*}$ & $0.28^{*}$ & $0.24^{*}$ \\
\hline
\end{tabular}

Table 3: Factor scores (mean \pm S.D.), internal reliabilities, and their inter-correlations of the Nightmare Experience Questionnaire. Note: \# $\mathrm{p}<0.05$ vs. Men; ${ }^{*}$ Significant correlation at $\mathrm{p}<0.05$; CI: Confidence Interval.

The second factor, Negative Emotion, was consistent with the nightmare-related emotion reported previously [27]. As nightmares are vivid and high-emotionally dysphoric dreams that awaken the individual from sleep [3,6], where fear, terror, or anxiety are predominant emotions [2], a factor like this one in our study might be important to evaluate the nightmare severity as suggested [27]. The positive correlation between the nightmare frequency and this factor score was also in line with previous results [43]. Indeed, it has shown that the heightened reactivity to nightmare at night increases the individual's risk for the elevated distress at waking and the subsequent 
psychopathology in life [44], which in turn induces more frequent nightmares [40]. Moreover, we have found that women scored significantly higher on this factor than men did which was in accordance with the finding that women experienced explicitly more emotions in their dreams $[45,46]$. Furthermore, there are genderrelated biological differences in the cerebral processing of emotions which might be linked to the better episodic memory of emotion in women [47]. This factor supports that nightmare is related to a personality trait of an intense reaction to emotional distress $[26,42,48]$. The heightened emotional distress at waking as an underlying pathological base might in turn be aggravated by the nightmare reported in the emotion-related disorders such as borderline personality disorder or substance abuse disorder [10-13].

The third factor, Meaning Interpretation, corresponded to a domain of "search of explanations for the nightmare" reported by Köthe \& Pietrowsky [28]. The nightmare frequency positively correlated with this factor was also in line with a previous report [28]. Women in our study also scored higher on this factor than men did, which was consistent with previous studies that women rated their nightmares as more vivid and meaningful $[4,26,48]$. Interestingly, women who frequently experienced nightmares often have higher levels of open-mindedness, sensitivity, vulnerability, creativity, and artistic ability [28,49]. Moreover, other literature supports its correlation with frequency and its women preponderance. For instance, women have more nightmares [7], score higher on neuroticism trait than men do [50], and people with a prominent neuroticism tend to seek the meanings of nightmare [28].

The fourth factor, Horrible Stimulation, corresponded to a differing or idiopathic content, and to a repetitive or recurrent content of the nightmare, which were often focused on a specific terrific or excited event as reported previously [44]. Most scenes appeared in nightmares resembled the terrifying activity or adventure described by patients suffering from PTSD or other disorders with traumatic experiences [51]. The scenes being similar to the flashbacks of trauma, often disrupted the sleep quality considerably, in return might re-exaggerate the symptoms in these patients [52,53], which supports the positive correlation found between the nightmare frequency and this factor score in our participants.

However, one might also bear in mind several limitations of the current study design. Firstly, our participants were college students, who were in a time period of significant life change and adjustment to situations of novel and stressful, which might increase the nightmare frequency [54]. Although we could not guarantee that all our college students were non-stressful, it is absolutely necessary to apply NEQ to other age groups. Secondly, there have been significant differences between the posttraumatic and non-traumatic nightmares [3], although we have excluded PTSD patients from our study, we failed to note whether our participants had any covert early traumatic experiences. A future study designed according to different lifeexperiences is also warranted.

Nevertheless, we have developed a structure-validated, 20-item NEQ with four distinct factors, and found their positive correlations with the nightmare frequency, which might help to characterize a nightmare in our daily lives and in clinics. For instance, the Physical Effect and Negative Emotion, which relate more to the distress of a nightmare, the Meaning Interpretation, which relates more to its realistic or optimistic reasoning, and the Horrible Stimulation, which connects to its traumatic flashbacks, might specifically help to measure the different aspects of a nightmare experience in normal children and adolescents, and in patients with cardiovascular or neuropsychiatric disorders. Moreover, women showed higher scores of Negative Emotion and Meaning Interpretation in the current study, which from a limited angle, might help to understand the emotional deregulation and the related coping styles at waking in female patients with borderline personality disorder or substance abuse disorder.

\section{Acknowledgements}

The study was supported by the grants from the Natural Science Foundation of China. Dr. W.Wang: 91132715.

\section{References}

1. American Psychiatric (2013) Association Diagnostic and Statistical Manual of Mental Disorders: DSM-5. Arlington: Amerc Publishing (fifth edition).

2. Zadra A, Pilon M, Donderi DC (2006) Variety and intensity of emotions in nightmares and bad dreams. J Nerv Ment Dis 194: 249-254.

3. Levin R1, Nielsen TA (2007) Disturbed dreaming, posttraumatic stress disorder, and affect distress: are view and neurocognitive model. Psychol Bull 133: 482-528.

4. Levin R (1994) Sleep and dreaming characteristics of frequent nightmare subjects in a university population. Dreaming 4: 127-137.

5. Levin R, Fireman G (2002) Nightmare prevalence, nightmare distress, and self-reported psychological disturbance. Sleep 25: 205-212.

6. Nielsen T, Levin R (2007) Nightmares: a new neurocognitive model. Sleep Med Rev 11: 295-310.

7. Schredl M, Reinhard I (2011) Gender differences in nightmare frequency: a meta-analysis. Sleep Med Rev 15: 115-121.

8. Mume CO (2009) Nightmare in schizophrenic and depressed patients. Eur J Psychiatry 23: 177-183.

9. Ä̈̈̈arg $\tilde{A} 1 / 4 \mathrm{n}$ MY, Cilli AS, Kara H, Tarhan N, Kincir F, et al. (1998) Repetitive and frightening dreams and suicidal behavior in patients with major depression. Compr Psychiatry 39: 198-202.

10. Cernovsky ZZ (1985) MMPI and nightmares in male alcoholics. Percept Mot Skills 61: 841-842.

11. Cernovsky ZZ (1986) MMPI and nightmare reports in women addicted to alcohol and other drugs. Percept Mot Skills 62: 717-718.

12. Selby EA (2013) Chronic sleep disturbances and borderline personality disorder symptoms. J Consult Clin Psychol 81: 941-947.

13. Semiz UB, Basoglu C, Ebrinc S, Cetin M (2008) Nightmare disorder, dream anxiety, and subjective sleep quality in patients with borderline personality disorder. Psychiatry Clin Neurosci 62: 48-55.

14. Agargunr MY, Kara H, Ozer OA, Selvi Y, Kiran U, et al. (2003) Nightmares and dissociative experiences: The key role of childhood traumatic events. Psychiatry Clin Neurosci 57: 575-579.

15. Watson D (2001) Dissociations of the night: individual differences in sleep-related experiences and their relation to dissociation and schizotypy. J Abnorm Psychol 110: 526-535.

16. Woodward SH, Arsenault NJ, Murray C, Bliwise DL (2000) Laboratory sleep correlates of nightmare complaint in PTSD inpatients. Biol Psychiatry 48: 1081-1087.

17. Mehl RC, O'Brien LM, Jones JH, Dreisbach JK, Mervis CB, et al. (2006) Correlates of sleep and pediatric bipolar disorder. Sleep 29: 193-197.

18. Nielsen TA, Laberge L, Paquet J, Tremblay RE, Vitaro F, et al. (2000) Development of disturbing dreams during adolescence and their relation to anxiety symptoms. Sleep 23: 727-736.

19. Harvey AG, Jones C, Schmidt DA (2003) Sleep and posttraumatic stress disorder: a review. Clin Psychol Rev 23: 377-407.

20. Mellman TA, David D, Kulick-Bell R, Hebding J, Nolan B (1995) Sleep disturbance and its relationship to psychiatric morbidity after Hurricane Andrew. Am J Psychiatry 152: 1659-1663. 
Citation: Chen W, Xu Y, Zhu M, Tang Y, Huang S, et al. (2014) Development of a structure-validated Nightmare Experience Questionnaire in Chinese University Students. J Psychiatry 17: 1000147. doi:10.4172/2378-5756.1000147

Page 6 of 6

21. Neylan TC, Marmar CR, Metzler TJ, Weiss DS, Zatzick DF, et al. (1998) Sleep disturbances in the Vietnam generation: findings from a nationally representative sample of male Vietnam veterans. Am J Psychiatry 155: 929-933.

22. Ohayon MM, Shapiro CM (2000) Sleep disturbances and psychiatric disorders associated with posttraumatic stress disorder in the general population. Compr Psychiatry 41: 469-478.

23. Krakow B, Schrader R, Tandberg D, Hollifield M, Koss MP, et al. (2002) Nightmare frequency in sexual assault survivors with PTSD. J Anxiety Disord 16: 175-190.

24. Sjöström N, Hetta J, Waern M (2009) Persistent nightmares are associated with repeat suicide attempt: a prospective study. Psychiatry Res 170: 208-211.

25. Li SX1, Lam SP, Yu MW, Zhang J, Wing YK (2010) Nocturnal sleep disturbances as a predictor of suicide attempts among psychiatric outpatients: a clinical, epidemiologic, prospective study. J Clin Psychiatry 71: $1440-1446$.

26. Belicki K (1992a) The relationship of nightmare frequency to nightmare suffering with implications for treatment and research. Dreaming 2: 143-148.

27. Roberts J, Lennings C (2006) Personality, psychopathology and nightmares in young people. Pers Individ Dif 41: 733-744.

28. Köthe M, Pietrowsky R (2001) Behavioral effects of nightmares and their correlations to personality patterns. Dreaming 11: 43-52.

29. Belicki K, Chambers E, Ogilvie R (1997) Sleep quality and nightmares. Sleep Res 26: 637.

30. Krakow B1, Kellner R, Pathak D, Lambert L (1995) Imagery rehearsal treatment for chronic nightmares. Behav Res Ther 33: 837-843.

31. Martínez M P, Miró E, Arriaza R (2005) Evaluation of the distress and effects caused by nightmares: A study of the psychometric properties of the nightmare distress questionnaire and the nightmare effects survey. Sleep Hypn 7: 29-41.

32. Schredl M (2003) Effects of state and trait factors on nightmare frequency. Eur Arch Psychiatry Clin Neurosci 253: 241-247.

33. Schredl M (2010) Nightmare frequency and nightmare topics in a representative German sample. Eur Arch Psychiatry Clin Neurosci 260: 565-570.

34. IBM SPSS (2009) PASW statistics 180 command syntax reference. Chicago, IL: SPSS Inc.

35. Arbuckle JL (2008) Amos 17.00. Crawfordville, FL: Amos Development Corporation.

36. Jöreskog KG, Sörbom D (1984) LISREL-VI user's guide. Mooresville, In: Scientific Software (third edition).

37. Bentler PM, Bonett DG (1980) Significance tests and goodness of fit in the analysis of covariance structures. Psychol Bull 88: 588-606.

38. Bentler PM (1990) Comparative fit indexes in structural models. Psychol Bull 107: 238-246.
39. Browne MW, Cudeck R (1993) Alternative ways of assessing model fit. In: KA Bollen, JS Long (eds) Testing structural equation models. Sage Newbury Park: 136-162.

40. Levin R, Nielsen T (2009) Nightmares, bad dreams, and emotion dysregulation: A review and new neurocognitive model of dreaming. Curr Dir Psychol Sci 18: 84-88.

41. Krakow B, Hollifield M, Schrader R, Koss M, Tandberg D, et al. (2000) A controlled study of imagery rehearsal for chronic nightmares in sexual assault survivors with PTSD: a preliminary report. J Trauma Stress 13: 589-609.

42. Blagrove M, Farmer L, Williams E (2004) The relationship of nightmare frequency and nightmare distress to well-being. J Sleep Res 13: 129-136.

43. van der Kolk B, Blitz R, Burr W, Sherry S, Hartmann E (1984) Nightmares and trauma: a comparison of nightmares after combat with lifelong nightmares in veterans. Am J Psychiatry 141: 187-190.

44. Levin R, Fireman G, Spendlove S, Pope A (2011) The relative contribution of affect load and affect distress as predictors of disturbed dreaming. Behav Sleep Med 9: 173-183.

45. Hall CS, van de Castle RL (1966) The content analysis of dreams. New York: Appleton-Century-Crofts.

46. Schredl M, Sahin V, Schäfer G (1998) Gender differences in dreams: Do they reflect gender differences in waking life? Pers Individ Dif 25: 433-442.

47. Bradley MM1, Codispoti M, Sabatinelli D, Lang PJ (2001) Emotion and motivation II: sex differences in picture processing. Emotion 1: 300-319.

48. Belicki K (1992) Nightmare frequency versus nightmare distress: relations to psychopathology and cognitive style. J Abnorm Psychol 101: 592-597.

49. Hartmann E, Elkin R, Garg M (1991) Personality and dreaming: the dreams of people with very thick or very thin boundaries. Dreaming 1: 311-324.

50. Rossier J, Aluja A, García LF, Angleitner A, De Pascalis V, et al. (2007) The cross-cultural generalizability of Zuckerman's alternative five-factor model of personality. J Pers Assess 89: 188-196.

51. Mellman TA, David D, Bustamante V, Torres J, Fins A (2001) Dreams in the acute aftermath of trauma and their relationship to PTSD. J Trauma Stress 14: 241-247.

52. Duval M, Zadra A (2010) Frequency and content of dreams associated with trauma. Sleep Med Clin 5: 249-260.

53. Nielsen T, Zadra A (2000) Dreaming disorders. In: MH Kryger, T Roth, W Dement (eds). Principles and Practice of Sleep Medicine. Elsevier BV: 753-772.

54. Wood JM, Bootzin RR, Rosenhan D, Nolen-Hoeksema S, Jourden F (1992) Effects of the 1989 San Francisco earthquake on frequency and content of nightmares. J Abnorm Psychol 101: 219-224. 